\title{
ESCLEROSIS INFLAMATORIA ORBITARIA Y SÍNDROME DE FIBROSIS MULTIFOCAL
}

\section{SCLEROTIC INFLAMMATION OF THE ORBIT AND MULTIFOCAL FIBROSCLEROSIS SYNDROME}

\author{
MATAIX B ${ }^{1}$, LÓPEZ-NAVARRETE E ${ }^{1}$, LÓPEZ-DOMÍNGUEZ M², ÁNGELES R ${ }^{2}$
}

\section{RESUMEN}

Caso clínico: Mujer de 52 años que consulta en nuestro Servicio por proptosis bilateral. La aparición de sintomatología aguda digestiva durante el ingreso para el estudio de la proptosis, condujo al diagnóstico de un cuadro de fibroesclerosis multifocal, con afectación simultánea de los tejidos orbitarios, grasa mesentérica y tronco de encéfalo.

Discusión: El pseudotumor esclerosante orbitario es una rara entidad clinicopatológica, cuyas características en la clínica y en las pruebas de imagen pueden ser similares a las del pseudotumor inflamatorio idiopático. El diagnóstico diferencial es importante porque ambos difieren tanto en su tratamiento como en su pronóstico.

Palabras clave: Proptosis, pseudotumor esclerosante, pseudotumor inflamatorio, mesenteritis esclerosante, fibroesclerosis mutifocal.

\begin{abstract}
Clinical case: A fifty-two-year-old woman consulted our department because of a bilateral proptosis. The appearance of acute symptoms suggestive of a digestive disorder during her admission to hospital for the study of the proptosis, led to a diagnosis of multifocal fibrosclerosis with simultaneous affliction of orbital tissue, mesenteric fat and the brain stem.

Discussion: Idiopathic Sclerotic Inflammation of the orbit is a rare pathological entity, with similar clinical and radiological characteristics to orbital pseudo-tumour. The differential diagnosis is important because both these conditions differ in treatment and prognosis (Arch Soc Esp Oftalmol 2008; 83: 263-266).

Key words: Proptosis, sclerotic inflammation of the orbit, inflammatory pseudotumour of the orbit, sclerosing mesenteritis, multifocal fibrosclerosis syndrome.
\end{abstract}

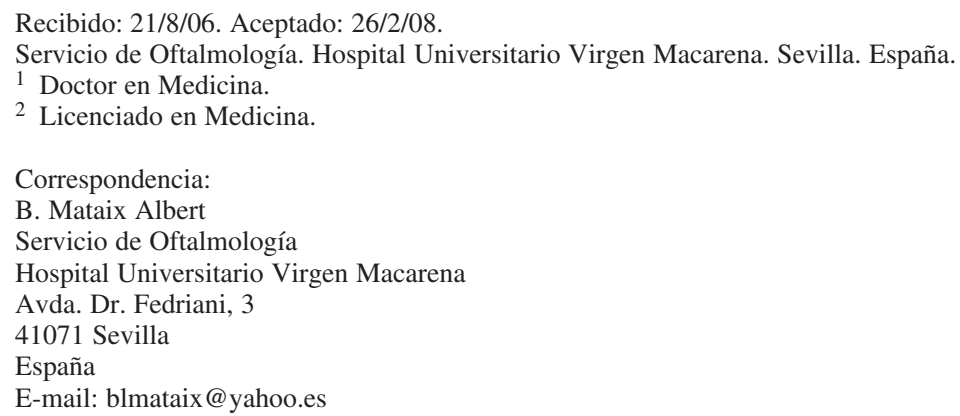




\section{INTRODUCCIÓN}

El pseudotumor esclerosante orbitario se manifiesta como una infiltración cicatricial, con efecto masa y signos inflamatorios crónicos progresivos determinados por la fibrosis (1).

En el caso que se presenta, junto a la baja prevalencia del citado pseudotumor, destaca la muy rara existencia conjunta de afectación del tronco del encéfalo y del mesenterio.

\section{CASO CLÍNICO}

Mujer de 52 años que consulta en nuestro Servicio por proptosis bilateral y disminución de agudeza visual (fig. 1).

En la exploración se observaba: exoftalmos bilateral simétrico con restricción de todos los movimientos extraoculares; leve hiperemia conjuntival en el segmento anterior y cierta borrosidad en el reborde papilar. La agudeza visual (AV) corregida era 0,2 en el ojo derecho y 0,1 en el izquierdo. Igualmente se detectó una percepción defectuosa del color rojo-verde.

La ecografía ocular mostraba una tumoración bilateral intraconal retroorbitaria de más de tres centímetros de diámetro que englobaba al nervio óptico. El servicio de radiología informó como diagnósticos más plausibles el linfoma y el pseudotumor inflamatorio idiopático. La Resonancia Magnética Nuclear (RMN) orbitaria confirmó los hallazgos ecográficos (fig. 2).

La paciente presentaba cefalea persistente, por lo que también se realizó una RMN craneal que evidenció una lesión extraaxial de $21 \mathrm{~mm}$ por delante del bulbo raquídeo (fig. 3). Se informó como una tumoración con una intensidad de señal muy similar a las lesiones orbitarias encontradas, lo que sugería

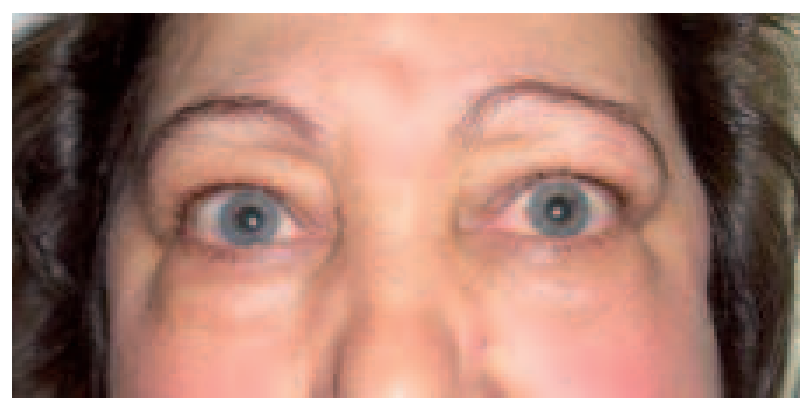

Fig. 1: Proptosis bilateral.

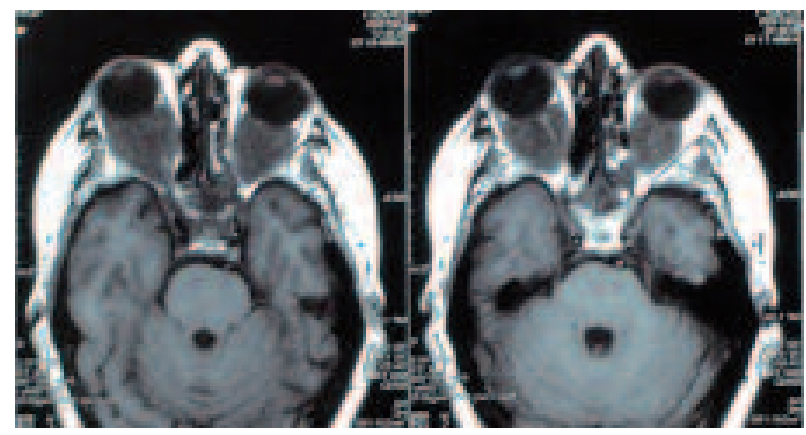

Fig. 2: RMN axial de órbitas en la que se observa tumoración intraconal bilateral englobando al nervio óptico.

la misma enfermedad. Ante la falta de otra focalidad neurológica, se mantuvo una actitud conservadora.

Las hormonas tiroideas, así como los marcadores $\mathrm{y}$ anticuerpos monoclonales fueron negativos.

La paciente fue hospitalizada para la realización de un tratamiento con corticoides, presentando durante su estancia un cuadro constitucional acompañado de epigastralgia. La Tomografía Axial Computerizada (TAC) abdominal mostró una masa englobando los vasos mesentéricos superiores (fig. 4). La posible existencia de un linfoma pospuso el tratamiento corticoideo.

La anatomía patológica de la lesión mesentérica se informó como inflamación esclerosante idiopática.

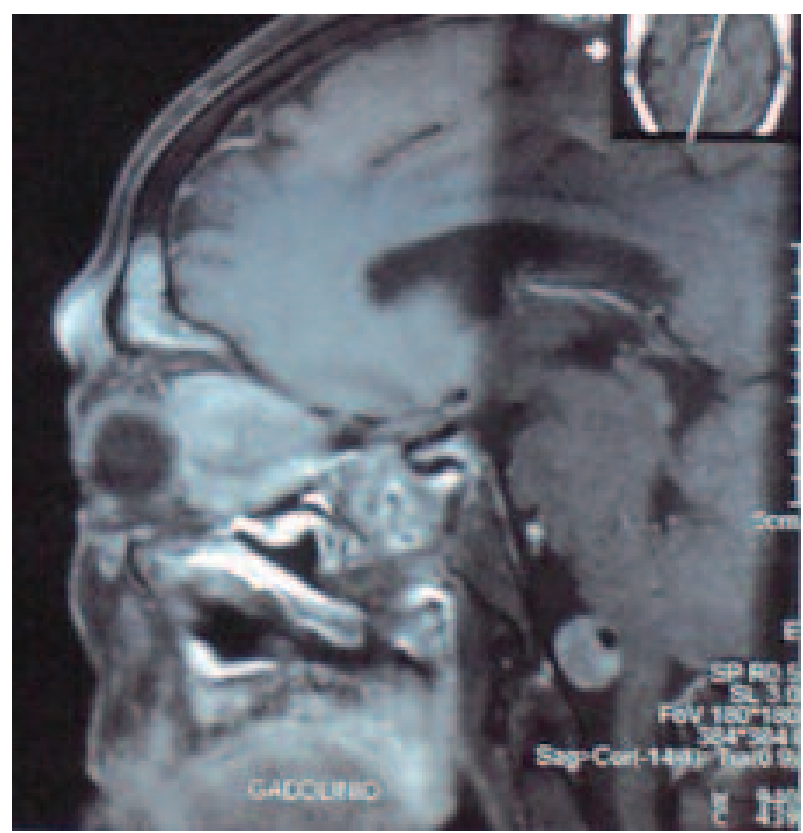

Fig. 3: RMN sagital de cráneo en la que se observa lesión extraaxial situada por delante de bulbo raquídeo. 

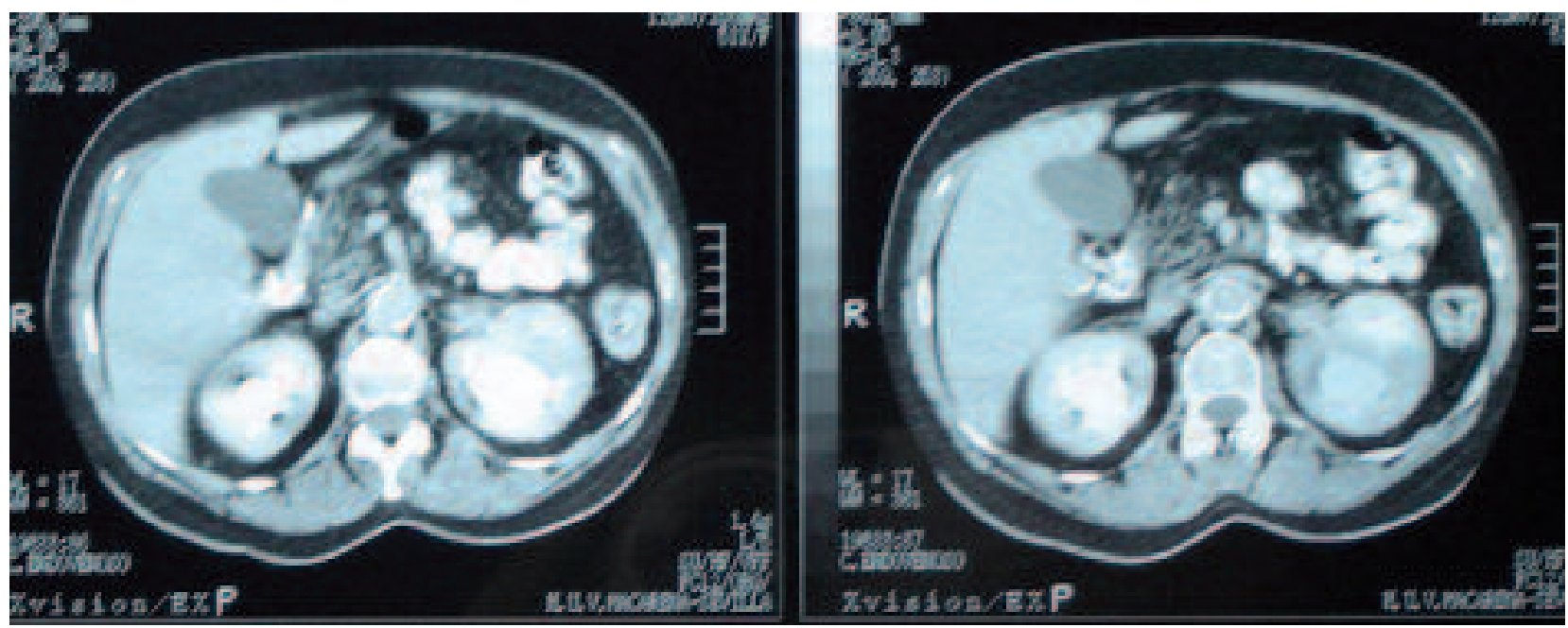

Fig. 4: TAC de abdomen que muestra masa englobando vasos mesentéricos superiores.

Durante el ingreso la agudeza visual empeoró hasta movimiento de manos en el ojo izquierdo, permaneciendo estable en el ojo derecho.

Con el diagnóstico de fibrosis mesentérica, se inició tratamiento con corticoides a dosis de 1 $\mathrm{mg} / \mathrm{kg} /$ día. Dada la respuesta favorable inicial, objetivada con la disminución del exoftalmos y mejoría de la agudeza visual (0,3 en ambos ojos), se decidió no utilizar inmunosupresores y continuar con el seguimiento en consultas externas.

Durante la evolución apareció la falta de respuesta al tratamiento corticoideo característico de la enfermedad, junto con una aguda e intensa afectación de la AV y campo visual bilaterales (percepción de bultos y DM: -8dB objetivada en la campimetría, respectivamente), con evidente sufrimiento del nervio óptico, que determinan la decisión de realizar una descompresión quirúrgica del canal orbitario. Ésta fue llevada a cabo vía transcraneal por el Servicio de Neurocirugía, el cual obtuvo una biopsia intraoperatoria.

La anatomía patológica se informó como inflamación esclerosante idiomática, confirmándose el cuadro de fibrosis multifocal.

En el postoperatorio hubo mejoría de la AV $(0,1$ en ambos ojos).

\section{DISCUSIÓN}

Como sugieren algunos autores (2), el pseudotumor esclerosante orbitario es una entidad diferente al pseudotumor inflamatorio, y deben tenerse en cuenta los siguientes aspectos: en primer lugar, conlleva pér- dida de la visión en unos meses tras el inicio de la fibrosis (1), segundo, se caracteriza por una mala respuesta al tratamiento corticoideo $(1,2)$, lo que nos obliga al conocimiento de otras posibles terapias, y en tercer lugar su posible asociación con otra afección sistémica (1) conformando un cuadro muy raro conocido como fibrosis multifocal. En total están descritos en la literatura 22 casos de inflamación esclerosante de la órbita con extensión extraorbitaria (3).

Aunque el diagnóstico de confirmación se realiza a partir de la anatomía patológica, tanto la exploración clínica como las pruebas de imagen nos pueden orientar hacia uno u otro diagnóstico (1). Así, la afectación unilateral habla a favor de un proceso inflamatorio y la bilateral de fibroesclerosis; en relación a las técnicas de imagen, en RMN T1 la lesión suele ser isointensa y en T2 hiperintensa en los procesos fibroesclerosantes, mientras que los pseudotumores inflamatorios en $\mathrm{T} 2$ son hipointensos (1).

Incluso la mala respuesta al tratamiento empírico con corticoides, en el caso de que se realizara para confirmar la afección inflamatoria, puede orientar hacia un proceso esclerosante (2).

Como tratamiento de la enfermedad esclerosante orbitaria está descrito el uso de corticoides y radioterapia aunque con pobres resultados. Kennerdall recomienda tratamiento combinado con cirugía, esteroides y radioterapia de forma temprana y agresiva. También se encuentra recogido en la literatura el uso de inmunosupresores como azatioprina y ciclofosfamida (3).

La paciente presentaba afectación del mesenterio junto a la afección orbitaria; es fácil comprender la 
importancia de este hallazgo pues la mesenteritis esclerosante coexiste con otros procesos de naturaleza maligna hasta en un 69\% (linfoma, melanoma y cánceres de mama, pulmón y colón) (4).

En este caso clínico destaca la importancia de diferenciar dos entidades clínicas independientes cuyo conocimiento nos puede permitir alterar la historia natural del paciente tanto a nivel oftalmológico como sistémico (5).

\section{BIBLIOGRAFÍA}

1. Schaffler GJ, Simbrunner J, Lechner H, Langmann G, Stammberger H, Beham A, et al. Idiopathic sclerotic inflammation of the orbit with left optic nerve compression in a patient with multifocal fibrosclerosis. AJNR Am J Neuroradiol 2000; 21: 194-197.

2. Pérez Moreiras JV, Prada Sánchez MC, Marín Valdés ME, Pérez Becerra E. Inflamaciones específicas y no específicas. Pseudotumores. In: Pérez Moreiras JV, Prada Sánchez MC. Patología Orbitaria. Barcelona: Edika Med; 2000; Tomo I: 155-219.

3. Zborowska B, Ghabrial R, Selva D, McCluskey P. Idiopahic orbital inflammation with extraorbital extension: case series and review. Eye 2006; 20: 107-113.

4. Horton KM, Lawler LP, Fishman EK. CT findings in sclerosing messenteritis (panniculitis): spectrum of disease. Radiographics 2003; 23: 1561-1567.

5. Hsuan JD, Selva D, McNab AA, Sullivan TJ, Saeed P, O'Donnell BA. Idiopathic sclerosing orbital inflammation. Arch Ophthalmol 2006; 124: 1244-1250. 